\title{
Proteogenomic Landscape of Gastric Adenocarcinoma Peritoneal Metastases
}

\section{Jaffer Ajani ( $\nabla_{\text {jajani@mdanderson.org ) }}$}

The University of Texas MD Anderson Cancer Center https://orcid.org/0000-0001-9946-0629

\section{Shuangtao Zhao}

UT MDACC

\section{Ruiping Wang}

The University of Texas MD Anderson Cancer Center

\section{Shumei Song}

Department of Gastrointestinal Medical Oncology, UT MD Anderson Cancer Center, Houston, TX, 77030 https://orcid.org/0000-0002-8128-6287

\section{Makoto Kobayashi}

The University of Texas MD Anderson Cancer Center

\section{Dapeng Hao}

The University of Texas MD Anderson Cancer Center

\section{Guangchun Han}

MD Anderson Cancer Center https://orcid.org/0000-0001-9277-2507

\section{Xingzhi Song}

The University of Texas MD Anderson Cancer Center

\section{Jianhua Zhang}

The University of Texas MD Anderson Cancer Center https://orcid.org/0000-0001-5412-9860

\section{Melissa Pizzi}

Department of Gastrointestinal Medical Oncology, UT MD Anderson Cancer Center, Houston, TX, 77030

\section{Namita Shanbhag}

Department of Gastrointestinal Medical Oncology, UT MD Anderson Cancer Center, Houston, TX, 77030

\section{Andrew Futreal}

The University of Texas MD Anderson Cancer Center https://orcid.org/0000-0001-8663-2671

\section{Brian Badgwell}

Department of Gastrointestinal Medical Oncology, UT MD Anderson Cancer Center, Houston, TX, 77030 https://orcid.org/0000-0002-9915-8355

\section{Kazuto Harada}

Department of Gastrointestinal Medical Oncology, UT MD Anderson Cancer Center, Houston, TX, 77030

\section{George Calin}

The University of Texas MD Anderson Cancer Center https://orcid.org/0000-0002-7427-0578 Jody Vykoukal 
The University of Texas MD Anderson Cancer Center

\section{Chuan-Yih Yu}

The University of Texas MD Anderson Cancer Center

\section{Hiroyuki Katayama}

The University of Texas MD Anderson Cancer Center https://orcid.org/0000-0003-3117-1390

\section{Samir Hanash}

The University of Texas MD Anderson Cancer Center https://orcid.org/0000-0002-4210-1593

\section{Linghua Wang}

The University of Texas MD Anderson Cancer Center https://orcid.org/0000-0001-9380-0266

\section{Article}

Keywords: Gastric adenocarcinoma (GAC), Proteomics, peritoneal carcinomatosis (PC)

Posted Date: October 5th, 2020

DOl: https://doi.org/10.21203/rs.3.rs-81037/v1

License: (c) (1) This work is licensed under a Creative Commons Attribution 4.0 International License. Read Full License 


\section{Abstract}

Advanced gastric adenocarcinoma (GAC) often leads to peritoneal carcinomatosis (PC) and is associated with very poor outcomes. Therapeutic are limited and effective. Here we report the first proteogenomic study of PC cells from a prospective GAC cohort ( $n=26$ patients). A total of 16,449 proteins were detected from whole cell extracts (TCEs). PC cells could be classified based on the protein profiles alone into three distinct unsupervised clusters (tumor cell enriched, immune cell enriched and tumor-immune mixed) that differed in expression of oncogene/immune-related proteins. Integrated analysis revealed enriched biological pathways and notably, novel druggable targets (cancer-testis antigens, kinases, and receptors) that could be exploited to develop effective therapies and/or tumor stratifications. Systematic comparison of expression levels of proteins and mRNAs revealed novel expression patterns of key cancer genes notably high mRNA and low protein expression of HAVCR2, low mRNA but high protein expression of cancer testis antigens CTAGE1 and CTNNA2. These results inform strategies to target GAC vulnerabilities.

\section{Introduction}

Gastric adenocarcinoma (GAC) is a common cancer with more than one million new cases each year. ${ }^{1}$ GAC is mostly diagnosed at an advanced stage. ${ }^{2}$ The median survival of patients with advanced GAC remains $<12$ months mainly because of the lack of effective therapies. ${ }^{2}$ Only one oncogenic driver-based approach (Her2-directed treatment with trastuzumab) has provided modest survival advantage ${ }^{3}$. Other therapeutic options (cytotoxic therapy or anti-angiogenic agents) are only transiently effective (exception is checkpoint inhibitor in patients with microsatellite instable GACs that are rare). GAC patients are prone to developing peritoneal carcinomatosis (PC) which can result in accumulation of ascites requiring repeated drainage, bowel/ureteral obstruction(s), cachexia, and rapid decline. PC occurs in $\sim 45 \%$ of GAC patients and often leads to $<6$-month survival. Intraperitoneal approaches have produced unsatisfactory results and there is no accepted standard approach. ${ }^{4}$

Molecular characterization of GACs in The Cancer Genome Atlas (TCGA) has provided valuable initial insights into GAC biology. ${ }^{5}$ However, all GACs analyzed were sourced from the primary GACs. Proteomics data was limited to $\sim 300$ protein using the reverse phase protein array (RPPA) platform, of which 45 proteins were associated with defined molecular subtypes. ${ }^{6}$ In general, advanced GAC has lagged in molecular investigations compared to other common cancer types. ${ }^{2}$ We previously reported on whole exome sequencing (WES) and bulk RNA sequencing of PC cells from patients with GAC. ${ }^{7}$ Proteomic profiling of advanced GACs provides further insights into molecular subtypes and potential treatment modalities.

In this study, we present a comprehensive unbiased proteomics profiling of PC cells from 26 advanced GACs using the liquid chromatograph mass spectrometry (LC-MS) technology. Global profiling of the total 
cell proteome of PC cells provides a rich resource for data mining and novel insights for therapeutic applications.

\section{Materials And Methods}

\section{Patient cohort and sample collection}

A total of 26 GAC patients with documented PC were selected for this study. All patients were treated at The University of Texas MD Anderson Cancer Center (Houston, USA). PC specimens were obtained during a therapeutic procedure once patients provided a written approved informed consent document under an Institutional Review Board approved protocol. PC is stage 4 cancer under the American Joint Committee on Cancer Staging Manual (8th edition). ${ }^{2,8}$ The clinical characteristics and treatment information of these patients are listed in the Supplementary Table-1. PC specimens were collected between February 2016 and March 2018 and the detailed information correlated with treatment were shown in the Fig. S1A.

\section{Generation of mass spectrometry based proteomic data and analysis}

Total cell extracts (TCEs) were obtained by sonication of cell lysate pellets in $4 \mathrm{M}$ Urea, $3 \%$ IsoPropanol, $20 \mathrm{mM}$ Tris containing the detergent $2 \%$ octyl-glucoside (OG, Sigma-Aldrich) and protease inhibitors (complete protease inhibitor cocktail, Roche Diagnostics) and phosphatase inhibitor (PhosSTOP, Roche Diagnostics) followed by centrifugation at $20,000 \times \mathrm{g}$ at $4{ }^{\circ} \mathrm{C}$ for $30 \mathrm{~min}$. TCE proteins were reduced in DTT and alkylated with acrylamide before fractionation of intact proteins using RP-HPLC. Proteins were desalted and separated using an off-line AQUITY UPLC Class-H system (WATERS) with reversed-phase column (4.6-mm internal diameter $\times 150-\mathrm{mm}$ length; Column Technology). Collected fractions were dried by lyophilization followed by in-solution digestion with trypsin (Mass Spectrometry Grade, Thermo Fisher).

A total of 23 fractions were subjected to LC-MS/MS analysis per ascites tumor cells. Tryptic peptides were injected onto reversed phase nanoLC-MS/MS using a nanoAquity LC system coupled online with SYNAPT G2-Si ion-mobility mass spectrometer (WATERS). Separations were performed using $75 \mu \mathrm{m}$ id $\times$ $360 \mu \mathrm{m}$ od $\times 25$-cm-long fused-silica capillary column (Column Technology) slurry packed with $3 \mu \mathrm{m}, 100$ $\mathrm{A}^{\circ}$ pore size $\mathrm{C} 18$ silica-bonded stationary phase. Following injection of $\sim 2 \mu \mathrm{g}$ of protein digest onto a C18 trap column (Waters, $180 \mu \mathrm{m}$ id $\times 20 \mathrm{~mm}$ ), peptides were eluted using a linear gradient of $0.35 \%$ mobile phase $B$ ( 0.1 formic acid in $A C N$ ) per minute for $90 \mathrm{~min}$, then to $95 \% \mathrm{~B}$ in an additional $10 \mathrm{~min}$, all at a constant flow rate of $300 \mathrm{~nL} / \mathrm{min}$.

LC-HDMSE Data was acquired in high resolution mode using Waters Masslynx (version 4.1, SCN 851). The capillary voltage was set to $2.80 \mathrm{kV}$, sampling cone voltage to $30 \mathrm{~V}$, source offset to $30 \mathrm{~V}$, and source temperature to $100^{\circ} \mathrm{C}$. Mobility utilized high-purity N2 as the drift gas in the IMS TriWave cell. Pressures in the helium cell, Trap cell, IMS TriWave cell, and Transfer cell were $4.50 \mathrm{mbar}, 2.47 \mathrm{e}-2 \mathrm{mbar}, 2.90 \mathrm{mbar}$, and 2.53e-3 mbar, respectively. IMS wave velocity was $600 \mathrm{~m} / \mathrm{s}$, helium cell DC was $50 \mathrm{~V}$, Trap DC bias 
was $45 \mathrm{~V}$, IMS TriWave DC bias was $3 \mathrm{~V}$, and IMS wave delay was 1,000 $\mu \mathrm{s}$. The mass spectrometer was operated in V-mode with a typical resolving power of at least 20,000. All analyses were performed using positive mode ESI using a NanoLockSpray source. The lock mass channel was sampled every $60 \mathrm{~s}$. The mass spectrometer was calibrated with a [Glu1] fibrinopeptide solution $(300 \mathrm{fmol} / \mu \mathrm{L})$ delivered through the reference sprayer of the NanoLockSpray source. Accurate mass LC-HDMSE data was collected in an alternating, low energy (MS) and high energy (MSE) mode of acquisition with mass scan range from $\mathrm{m} / \mathrm{z}$ 50 to 1800 . The spectral acquisition time in each mode was $1.0 \mathrm{~s}$ with a 0.1 -s inter-scan delay. Data were collected in the low energy HDMS mode, at constant collision energy of $2 \mathrm{eV}$ in both Trap cell and Transfer cell. In high energy HDMSE mode, the collision energy was ramped from 25 to $55 \mathrm{eV}$ in the Transfer cell only. The RF applied to the quadrupole mass analyzer was adjusted such that ions from $\mathrm{m} / \mathrm{z}$ 300 to 2,000 were efficiently transmitted, ensuring that any ions observed in the LC-HDMSE data less than $\mathrm{m} / \mathrm{z} 300$ were known to arise from dissociations in the Transfer collision cell.

The acquired LC-HDMSE data were processed and searched against the Uniprot proteome database (Human, January 2017) through ProteinLynx Global Server (PLGS, Waters Company) with two trypsin miss cleavage allowed. The modification search settings included cysteine (Cys) alkylation with acrylamide (71.03714@C) as a fixed modification, and methionine (Met) oxidation (15.99491@M) as a variable modification. The searched data was filtered with False Discovery Rate $4 \%$. The spectral counts for each protein driven by the identified peptides was used as a measure of protein abundance. The normalization was done by each protein spectral counts divided by the total spectral counts of each patient ascites cells multiplied by the factor 50,000 .

\section{Proteomic data processed for TCEs}

Proteins expression values in all samples were generated with the total peptides aggregated by the same gene symbol in TCE platform. Proteins which were not identified in some samples were assigned a value of 0 . All values of proteins were then log2-transformed for further downstream processes. Highly expressed proteins were defined using a cut off of total peptides of $>50$ (Fig. S1B-C).

\section{Sample classification based on proteomic data}

A total of 1,980 out of 16,449 proteins detected in $\geq 80 \%$ of all samples $(n=26)$, were selected for principal component analysis (PCA) to assess the distribution of samples based on the global protein expression profiles, explore the optimal three clusters (tumor cell enriched, immune cell enriched and tumor immune mixed) and evaluate the possible batch effects (Figs. 1B-D; Figs. S2A-B). PCA analysis using TCE proteins indicated minimal batch effects and all samples were included for subsequent analyses. Then 2,361 differentially expressed proteins (DEPs) were identified from all proteins $(n=$ 16,449) among three clusters defined by PCA and used for the unsupervised hierarchical clustering analysis. Protein expression data in normal gastric tissues were downloaded from the Human Protein Atlas (HPA) and used as a control to select tumor specific proteins (Fig. S1E). Group enriched proteins were selected by applying a cut-off of expression fold change $\geq 2$ and adjusted $p$ value (FDR q-value) < 0.05 . 


\section{Pathway enrichment analysis}

Gene sets of interest were processed using Gene Set Enrichment Analysis (GSEA) ${ }^{9}$ against the curated gene sets ( $\mathrm{H}$ and $\mathrm{C} 2$ collections) downloaded from Molecular Signature Database (MSigDB) ${ }^{10}$ to identify the significantly enriched signaling pathways (criteria: FDR $<0.01$ and overlapped genes numbers $\geq 3$ ). The results were subsequently visualized in $\mathrm{R}$ with ggplot2 package.

\section{DNA and RNA data analysis}

Whole-exome sequencing (WES) was performed on 22 of 26 PC specimens, with 11 having matched gDNAs from peripheral blood. RNA sequencing was performed on 21 specimens for which MD data were available. DNA and RNA extractions, sequencing, data processing, quality check, and analysis were performed as previously described. ${ }^{7}$

\section{mRNA and protein correlation analysis}

To assess the correlation between mRNA and protein abundance, the overlapping genes $(n=1016)$ expressed in $\geq 95 \%$ samples that were common between the two platforms for 21 samples were subjected to Pearson correlation analysis.

\section{Statistical analysis}

The statistical methods used in this study were performed in the R statistical environment (v3.5.3). The evaluation of normal distribution was checked using the Shapiro-Wilk test before different expression analysis. The statistical significance of differences observed among clusters was determined by the ANOVA test or $t$ test for normal distribution data and the non-parametric Kruskal-Wallis test or Wilcoxon test for non-normal distribution data when comparing continuous variables, and the Fisher's Exact test when comparing frequencies of clinical factors. To control for false discovery rate (FDR) and correct $\mathrm{p}$ values from multiple testing, the Benjamini-Hochberg method was applied to calculate an FDR adjusted $\mathrm{p}$-value (or q-value). The Kaplan-Meier plots and log-rank tests were used for univariate survival analysis among three clusters. Pearson's correlation coefficient was calculated to evaluate the association between two continuous variables. Hypothesis testing was performed in a two-sided manner, with $\mathrm{p}$-value or adjusted $p$ value (if applicable) $<0.05$ considered to be statistically significant.

\section{Results}

\section{The proteomics landscape of PC cells}

LC-MS was performed on PC specimens from 26 patients (Fig. 1A, left). Table S1 summarizes the clinical and pathological characteristics of patients included in this study. PCA indicated minimal batch effects (Fig. S1D), and all samples passed quality filtering and were included in subsequent analyses. A total of 16,449 proteins were detected in one or more samples (Fig. 1A, middle), of which 3,661 TCE proteins were highly expressed in TCE (Fig. 1A, right). Substantial variation in protein abundance was observed 
between cell populations. A total of 637 TCE proteins were highly abundant and detected in all 26 samples whereas 2,273 proteins were detected with 0 expression value. Some of the GAC-associated and functionally well characterized proteins such as EGFR, ERBB2, YAP1, SOX9, TP53, and CDH1, exhibited variable abundance (Fig. 1B). The mean value of proteins in each patient was similar with median (Fig. 1C) between adenocarcinoma subtypes (NOS and SRC) and histology classifications (diffuse and intestinal). Especially, the proteins distribution and family construction were balanced between NOS and $\operatorname{SRC}(p=0.527$, Fig. 1D) but for histological subtypes (Fig.S1F).

\section{Proteomic profiling classified PC specimens into 3 groups with differential tumor cellularity}

To profile the global proteomic patterns of PC cells, we performed unsupervised hierarchical clustering analysis of TCE proteins which revealed 3 subgroups (Fig. 2A, Figs.S2A-B). Further investigations of the canonical marker (lineage-related and oncogenic) gene expression and differentially expressed proteins (DEPs, Table S2) suggested great variation in tumor cell compositions (Fig. 2A, top). For example, PC specimens in the Cluster A showed the highest expression of the epithelial lineage markers EPCAM and KRT8, and GAC driver genes CDH1, YAP1 and ERBB2, while exhibited the lowest expression of PTPRC, the immune cell marker, which together indicates that Cluster A was predominantly enriched with tumor cells. Similarly, PC specimens in Cluster $\mathrm{C}$ showed the highest expression of the immune cell marker PTPRC and exhibited low or no expression of the epithelial lineage markers or GAC related oncogenes, indicating abundant immune cells. Cluster B, however, showed mixed expression features of both epithelial and immune cells, and was therefore classified as "tumor-immune mixed".

Whole-exome sequencing (WES) data was generated for 22 of 26 samples and all the 22 samples were with matched germline DNAs (Table S1). Somatic mutation analysis identified frequent mutations in $C D H 1, T P 53, K M T 2 C$, with frequencies that were similar to those reported in 34 independent samples in our recent study ${ }^{7}$ (Fig. 2B). We next integrated the mutational profiles and proteomic data which strongly supported the proteomics-based cluster designation. We observed a greater number of somatic mutations in GAC driver genes and a higher mutation burden (TMB) in samples in Cluster A (tumor cell enriched), whereas no somatic mutations were detected in Cluster $C$ (immune cell enriched) (Figs. 2B\&D). Concordantly Cluster A samples exhibited extensive copy number alterations while no alterations were detected in Cluster C (Fig. 2C). Overall, the genomic data recapitulated features of the proteomics-based classification. These results demonstrate a high degree of proteomic inter-patient heterogeneity resulting from differential tumor cell compositions.

We further examined the clinical relevance of proteomics-based classification. Patients' age was significantly higher in clusters $\mathrm{A} / \mathrm{B}$ than in cluster $\mathrm{C}$ (the Fisher exact test $p=0.037$, Fig.S2C), but it could not independently affect the clusters divided by the significant proteins (Fig. S2D). In addition, the frequency of the signet ring cell carcinoma (SRC) was higher in clusters A and B compared with cluster C, although it did not reach statistical significance (Fig. 2E). We also observed a trend of shortened survival 
in patients in cluster $A$ (Fig. $2 F$, log-rank $P=0.07)$. These results indicate differences in clinical and histopathological phenotypes between proteomics-stratified groups.

\section{Dysregulated molecular pathways and potential druggable targets in PC cells of Cluster A.}

A great number of upregulated proteins were identified in tumor cells of Cluster A from DEP analysis of TCE data. To further understand the molecular consequences of these highly abundant proteins, we performed the pathway enrichment analysis to investigate dysregulated molecular processes informed by the proteomics data (see Methods). A total of 62 upregulated Hallmark and KEGG pathways were identified which were predominantly composed of oncogenic and metabolic signaling (Fig. 3A), such as glycolysis, oxidative phosphorylation (OXPHOS), mTORC1 signaling, MYC, TP53 and, TGF-beta pathways (Fig. 3B). These results are in line with the high tumor cell contents of PC specimens in Cluster A.

We next sought to identify tumor-cell specific protein targets in PC cells in Cluster A. We applied a stepwise filtering process to identify TCE proteins that were tumor specific, highly abundant and significantly enriched in PC cells of Cluster A (Fig. 3C). Consequently, 560 TCE proteins were identified. Among them, 68 TCE proteins were annotated as functionally important (Fig. 3D) which included 3 DEPs (EGFR, ERBB2, CDK4) as actionable targets with FDA approved inhibitors, 1 DEP (PAK4) is an emerging and attractive target and has been reported involving cytoskeleton remodeling, invasion and metastasis ${ }^{11}$ and 2 DEPs (CTAGE1 and CTNNA2) are cancer testis antigens and potential targets for cancer immunotherapy, especially for CTNNA2 with significantly high expression value of proteins/mRNAs in SRC compared to NOS subtype (Fig. S3). High expression of these DEPs in PC cells in Cluster A was evident in the TCE data (Fig. 3E).

We furtherly explored the proteomic characteristics between NOS and SRC in Cluster A. And we discovered that some critical genes were significantly upregulated in the SRC subtype compared to NOS, which played a positive role in the metabolic, cell cycle, and oncogenic (PTPRC, PYCARD) pathways (Fig. 3F). A total of 7 upregulated Hallmark and KEGG pathways were identified to be correlated with metabolic and cell cycle pathways in both two histological types, but with an oncogenic pathway (TNF signaling pathway) in SRC (Fig. 3G). Collectively, the two subtypes in Cluster A were with same biological functions in PC.

\section{Dysregulated molecular pathways and potential druggable targets in PC cells of Cluster C.}

Using similar approaches, we analyzed PC cells of cluster C. A total of 35 upregulated pathways were identified which are predominantly immune related (Fig. 4A-B), such as neutrophil degranulation, neutrophil activation, neutrophil-mediated immunity, and cytokine production. These results were in line with immune cell enriched profile of samples in Cluster $C$. We next identified immune-cell specific protein targets in PC cells of Cluster C. A total of 2,361 TCE DEPs were identified. Forty TCE proteins were annotated as functionally important (Fig. 4C-D). Among them, 6 DEPs (HAVCR2, CEACAM1, ITGAM, C10orf54, LTF and S100A12) are emerging and attractive targets for cancer immunotherapy. High expression of these DEPs in PC cells of Cluster C were mainly identified by the protein analysis. 
Furtherly, we performed the adenocarcinoma subtypes analysis in the clusters B and C (Fig.S2E). The DEGs result showed that the upregulated genes in the SRC subtype patients were enriched in the oncogenic (RAC1, PTPN11, DUT), metabolic and cell cycle pathways, but the immune correlated pathways in the NOS subtype (Fig. 4E). This was in line with the GSEA results between NOS and SRC subtypes (Fig. 4F). All the results above suggested that the adenocarcinoma subtypes could help distinguish the patients with favorable prognosis from the clusters $\mathrm{B}$ and $\mathrm{C}$.

\section{Proteomic features associated with somatic mutations of CDH1, TP53 and KMT2C}

$\mathrm{CDH} 1, \mathrm{TP} 53$, and KMT2C are frequently mutated in advanced GAC. However, these genomic alterations are not directly druggable. To identify potential actionable targets in tumor cells with somatic alterations (loss of function) in CDH1, TP53, or KMT2C, we performed DEP and pathway enrichment analyses. Samples were stratified into two groups (high or low) based on their corresponding TCE protein expression levels (Fig. 5A). Interestingly, proteins that were significantly upregulated in the CDH1-high PC cells included ERBB2, EGFR, CTNNA2, and SOX9, three of which are druggable. IGF2R and CREBBP were significantly elevated in tumor cells in the TP53-low group, but no potential targets were detected from the KMT2C stratified groups. Further, the pathway analysis identified upregulated OXPHOS and cell cycle signaling in $\mathrm{CDH} 1$-high PC cells and both pathways are druggable (Fig. 5B). Collectively, these data provide potentially druggable targets which could not be obtained from mutation data and related pathways.

\section{Correlation between mRNA and protein expression and key marker genes}

To assess the complementary value of proteomic and mRNA data, a correlation analysis was carried out that involved 6,638 proteins and mRNAs divided into four quantiles (i.e., Q1, Q2, Q3, and Q4) based on their overall distribution. Protein abundances showed different correlation with their correspondent mRNAs in different quantiles (Fig. 6A). We assessed the relationship between the protein and mRNA expressions in the consistent and inconsistent quantile district, along with the protein functional categories and pathway enrichment analysis. Among the consistently high genes in Q4 $(n=860)$, we identified enzymes and transporters as the top two families, and oncogenic signaling and metabolic signaling as the significantly enriched pathways. Importantly, the oncogene FN1 was correlated with the EMT signaling pathway. However, within the consistently low genes in Q1 ( $n=741)$, we identified enzymes and transcriptional factors as another top two families, and obtained dominantly cell cyclerelated processes (Fig. 6B). Within the inconsistent expression genes in Q1 and Q3/4, we similarly obtained the top two families and the functional pathways except for proliferation-related processes (DNA repair and apoptosis), including potentially interesting therapeutic targets such as cancer/testis proteins (CTNNA2, LTF, CTAGE1), and immunotherapy targets such as LAIR1 and HAVCR2 (Fig. 6C). Thus, the genes with consistent or inconsistent expression patterns between mRNA and protein were important to study PC metabolomics, especially the highly expressed ones were involved in the key signaling 
pathways in PC. Together, these data suggested that the protein data provided complementary information to yield an improved understanding of biological processes involved in PC.

\section{Discussion}

When the point and structural mutations, along with epigenomic alterations accumulate enough to dysregulate the transcriptome, it finally leads to proteome dysregulation contributed to cancer occurrence. ${ }^{12}$ Proteins are considered as the most abundant class of functional molecules in the cell, and the central dogma guides that the information flows from genome and epigenome to the proteome. We therefore studied a unique cohort of GAC patients with PC. We report a proteogenomic landscape of 26 PC specimens, using the cutting-edge technology, mass spectrometry, to get a proteomics read out of TCEs. Our results provide complimentary information to TCGA-affiliated CPTAC project ${ }^{13-15}$ and a panoramic view of altered cancer proteome including signaling pathways in PC cells.

We used molecular classification to stratify patients into subtypes correlated with prognosis and biological characteristics. The proteomic analysis within 26 PC specimens resulted into three clusters, indicating heterogeneity ingrained in GAC PC cells. Meanwhile, a considerable number of genes were identified with mutations and insertions/deletions in three clusters, especially there was abundance of more mutations and deletions in clusters A and B. Importantly, the signaling pathways altered distinctively in the proteomic subtypes by cellular processes enrichment. The oncogenic signaling pathways (TGF $\beta$ and MTORC1) and metabolic signaling pathway (Glycolysis and OXPHOS) dominated cluster $A$, but immune-related pathways were enriched in cluster $C$. Meanwhile some targetable oncogenes such as CTAGE1, CTNNA2, ERBB2, and EGFR, were significant more enriched in cluster A, and some immune-related specific proteins were identified such as C10orf54 (VISTA), ITGAM (CD11B), LAIR1, HAVCR2 (TIM3) and LTF. The adenocarcinoma subtypes were with the similar protenomic biological functions in the Cluster A. However, the SRC subtype was predominantly enriched with oncogenic, metabolic, and cell cycle pathways in the Clusters B and $C$ but immune response in NOS subtype. These data suggest that patients in cluster A could benefit from targeting cancer cells (specific alterations in the DNA) but patients in cluster $\mathrm{C}$ might benefit from targeting immune cells, however, more studies are required.

The proteogenomic analysis within three genes (CDH1, TP53 and KMT2C) revealed a poor correlation between genomic and proteomic data, which was observed similarly in the CPTAC projects. ${ }^{13-15}$ The discordance between gene mutations and protein abundance might be due to tumor heterogeneity and/or limited number of samples with DNA mutations to calculate the correlation. This discrepancy might have been caused by the study of low number of PC cancer cells with DNA mutations while the protein abundance results might have been influenced by the neighboring cells including cells without alterations in these genes. We detected a high frequency of mutations in these CDH1, TP53 and KMT2C genes and corresponding proteomic changes. For example, the correlation between loss-of-function $\mathrm{CDH} 1$ mutation led to upregulation of several key genes such as ERBB2, EGFR, SOX9 and CTNNA2 suggesting 
establishing a loss-of-function of tumor suppressor gene might afford opportunities to target oncoproteins for advantage.

It is understood that DNA mutations/alterations are reflected in proteins but in some instances, proteins are further processed and modified, thus proteins reflect the final functional product in cells. ${ }^{16}$ Therefore, some of the targets warrant further investigation, especially for the unique proteins/peptides in PC cell that are not abundant or absent in normal cells.

Furthermore, correlating proteins with the corresponding mRNAs from the same PC specimen would support active biogenesis of protein products by PC cells validating it as a potential target. Detection of mRNA or protein may not always be meaningful, for example, we noted that while many mRNAs corresponded to high transcription of (Qs3/4) genes (i.e., HAVCR2) but had low expression (Q1) of protein and sometimes mRNAs with low expression values (Q1) were associated with high expression values (Qs3/4) of protein, including several important molecules we noted (i.e., CTNNA2, LTF, CTAGE1 and LAIR1). Thus, it may be that when mRNA and corresponding protein are over-expressed, target validation by functional studies should be considered. However, proteins expressed in different quantiles may also of importance to cancer cells.

In summary, our integrative proteogenomic characterization revealed new therapeutic targets in signaling proteins, metabolic enzymes, kinases, and cancer antigens for treating (upon functional validation) PC. Our study provides a rich resource characterizing proteomics and proteogenomics of PC cells. Currently, proteomics analysis of GAC is quite limited and that of PC cells almost non-existent. Therefore, our novel findings suggest that some of the newly discovered targets warrant further assessments.

\section{Declarations}

\section{Acknowledgements}

The authors appreciate all the gastric cancer patients and their families for their help with the donation of the specimens for this study.

\section{Contributors}

JAA and LW conceived and jointly supervised the study. STZ conducted the major bioinformatics and biostatistics analysis of the multiplatform data, produced all the figures and tables for this manuscript. RW assisted with WES analysis, DH assisted with RNA-seq analysis and GH assisted with MS data analysis. JZ and XS contributed to raw WES data processing. SS, MZ, MK and MPP assisted with clinical samples consent, collection, pathological evaluation and MS data generation. $\mathrm{KH}$ took charge of ascites cells isolations, DNA/RNA extraction. AF help to revise the manuscript.

\section{Funding}


This study was supported in part by the National Cancer Institute awards CA129906, CA127672, CA138671 and CA172741 and the DOD grants: CA150334 and CA162445 to JAA and DOD grants CA160433 and CA170906 to SS, and the start-up research funds provided to LW by the Department of Genomic Medicine, Division of Cancer Medicine of MD Anderson Cancer Center. This study was also supported by SMF Core grant CA016672 (SMF).

Also supported by Stupid Strong Foundation and V Foundation

\section{Competing interests}

The authors declare that they have no competing interests.

\section{Patient consent for publication}

Not required.

\section{Ethics approval}

This project was approved by the institutional review committee.

\section{Provenance and peer review}

Not commissioned; externally peer reviewed.

\section{Data available}

All WES and RNA-Seq data have been deposited at the European Genome-phenome Archive (EGA). The datasets can be fully accessed under the accession number EGAS00001003180. Further information about EGA can be found on https://ega-archive.org ('the EGA of human data consented for biomedical research': http://www.nature.com/ng/journal/v47/n7/full/ng.3312.html). The MS data in the current study are available from the corresponding author upon a reasonable request.

\section{References}

1. Bray F, Ferlay J, Soerjomataram I, Siegel RL, Torre LA, Jemal A. Global cancer statistics 2018 : GLOBOCAN estimates of incidence and mortality worldwide for 36 cancers in 185 countries. CA Cancer J Clin 2018;68:394-424.

2. Ajani JA, Lee J, Sano T, Janjigian YY, Fan D, Song S. Gastric adenocarcinoma. Nat Rev Dis Primers 2017;3:17036.

3. Bang YJ, Van Cutsem E, Feyereislova A, et al. Trastuzumab in combination with chemotherapy versus chemotherapy alone for treatment of HER2-positive advanced gastric or gastro-oesophageal junction cancer (ToGA): a phase 3, open-label, randomised controlled trial. Lancet 2010;376:687-97. 
4. Macri A, Morabito $F$. The use of intraperitoneal chemotherapy for gastric malignancies. Expert Rev Anticancer Ther 2019;19:879-88.

5. Cancer Genome Atlas Research N. Comprehensive molecular characterization of gastric adenocarcinoma. Nature 2014;513:202-9.

6. Creighton CJ, Huang S. Reverse phase protein arrays in signaling pathways: a data integration perspective. Drug design, development and therapy 2015;9:3519.

7. Wang R, Song S, Harada K, et al. Multiplex profiling of peritoneal metastases from gastric adenocarcinoma identified novel targets and molecular subtypes that predict treatment response. Gut 2020;69:18-31.

8. Siegel RL, Miller KD, Jemal A. Cancer statistics, 2018. CA Cancer J Clin 2018;68:7-30.

9. Subramanian A, Tamayo P, Mootha VK, et al. Gene set enrichment analysis: a knowledge-based approach for interpreting genome-wide expression profiles. Proc Natl Acad Sci U S A 2005;102:15545-50.

10. Liberzon A, Birger C, Thorvaldsdottir H, Ghandi M, Mesirov JP, Tamayo P. The Molecular Signatures Database (MSigDB) hallmark gene set collection. Cell Syst 2015;1:417-25.

11. Murray BW, Guo C, Piraino J, et al. Small-molecule p21-activated kinase inhibitor PF-3758309 is a potent inhibitor of oncogenic signaling and tumor growth. Proceedings of the National Academy of Sciences 2010;107:9446-51.

12. Hanahan D, Weinberg RA. Hallmarks of cancer: the next generation. Cell 2011;144:646-74.

13. Zhang B, Wang J, Wang $X$, et al. Proteogenomic characterization of human colon and rectal cancer. Nature 2014;513:382-7.

14. Zhang H, Liu T, Zhang Z, et al. Integrated Proteogenomic Characterization of Human High-Grade Serous Ovarian Cancer. Cell 2016;166:755-65.

15. Mertins P, Mani DR, Ruggles KV, et al. Proteogenomics connects somatic mutations to signalling in breast cancer. Nature 2016;534:55-62.

16. Ge S, Xia X, Ding C, et al. A proteomic landscape of diffuse-type gastric cancer. Nat Commun 2018;9:1012.

\section{Figures}


B
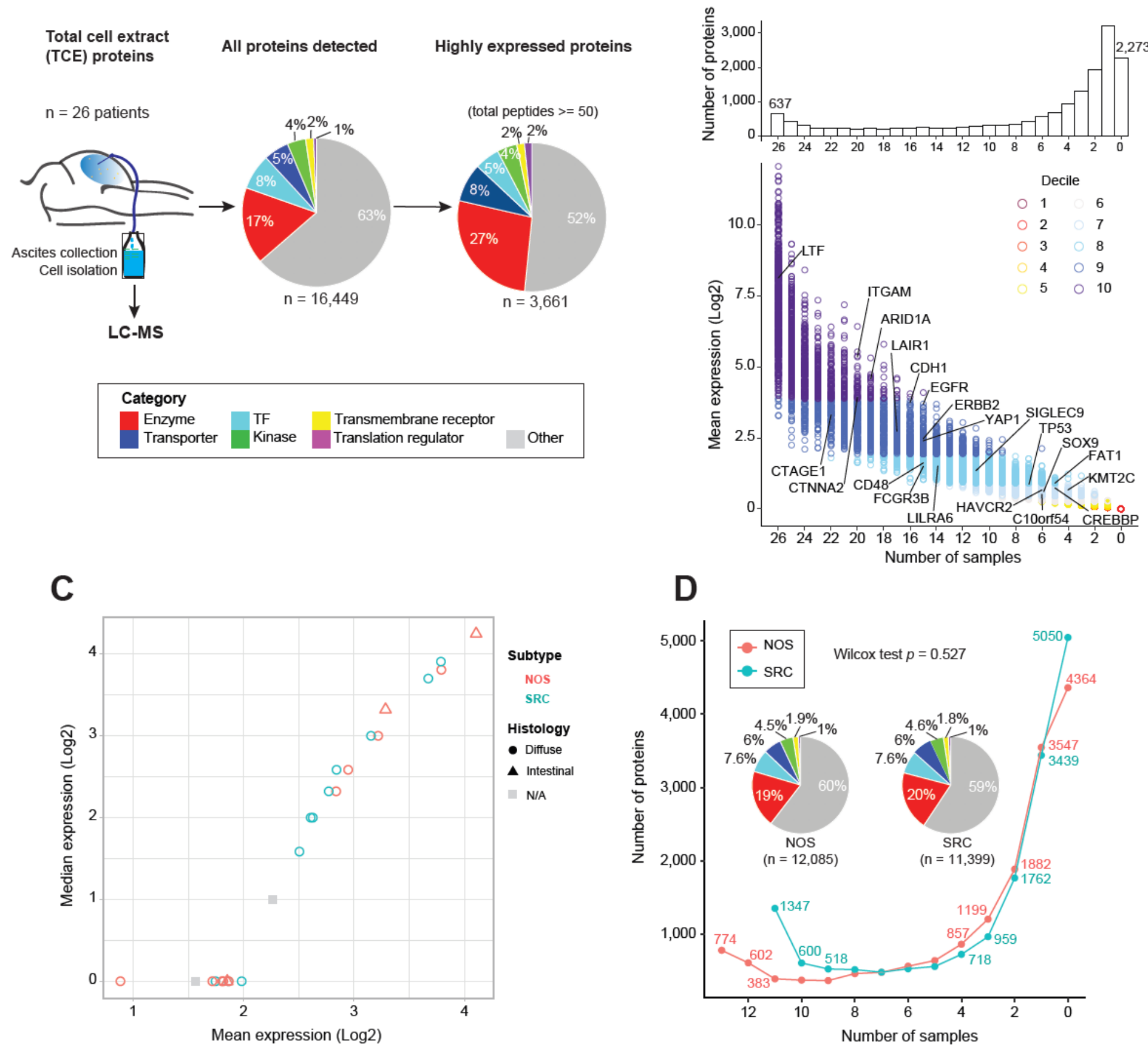

\section{Figure 1}

A landscape of the mass spectrometric-derived cell from 26 advanced gastric cancer patients with peritoneal metastases. From each patient, the ascites was collected and from which the cells were isolated and processed for liquid chromatography-mass spectrometry (LC-MS). A) A schematic overview of the protein repertoires from total cell extract (TCE). The total number of proteins detected (total peptides $>0$ ) by each approach, the highly expressed proteins (total peptides $₫ 50$ ), and the proportion of proteins unique to each approach or detected by TCE. The protein categories are annotated in the pie charts based on their molecular functions. TCE, protein detected from total cell extract; TF, transcription 
factor. B) Distribution of protein quantitation measured as mean intensity (Log2 scaled) by the number of samples they are detected in, color coded by the decile. The bar plots on the top display the total counts of proteins quantified across various number of samples. The value 0 stands for the number of proteins that were not detected in any of the samples analyzed. Some representative genes are labelled on the plots. C) Scatter plot shows the expression distribution with mean and median values between adenocarcinoma subtypes and histology classifications. D) Cumulative number of proteins identified as a function of the patient numbers. Proteins family was shown with pipe plots between NOS and SRC. Statistical method: wilcox test. 
A

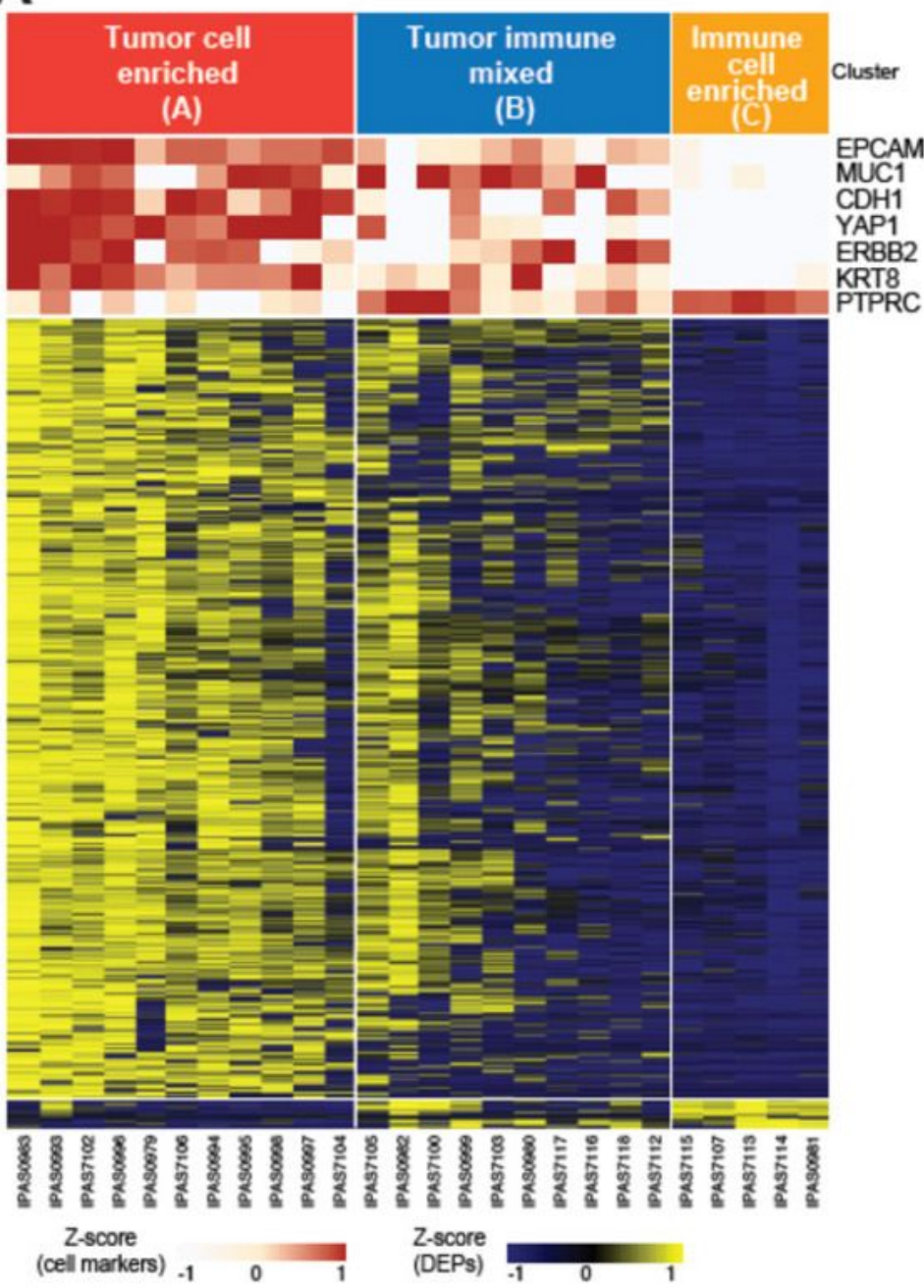

C

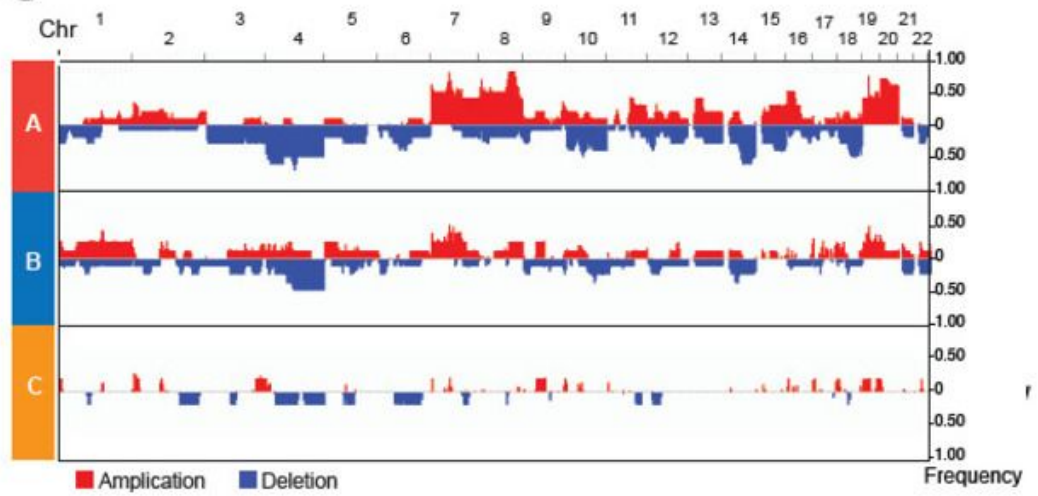

B

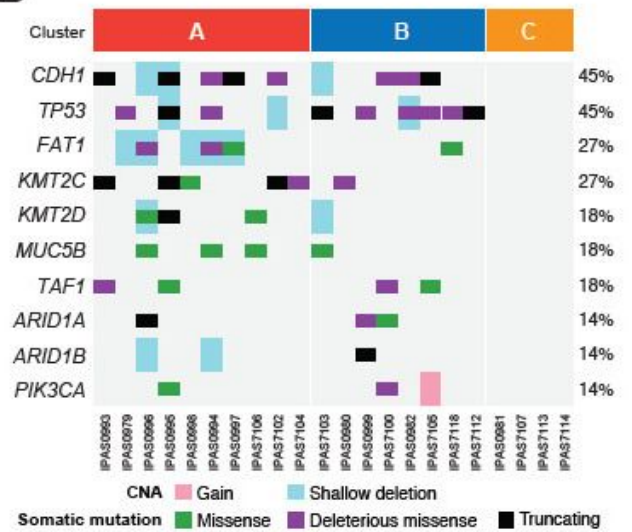

D

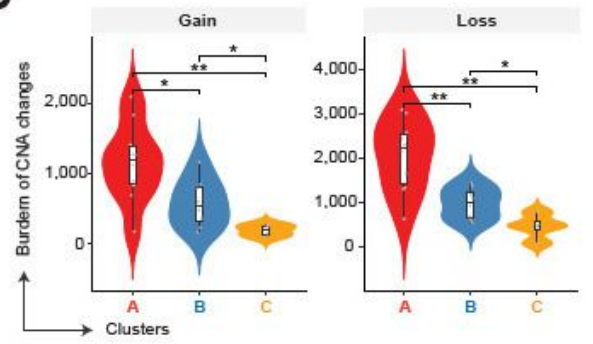

E

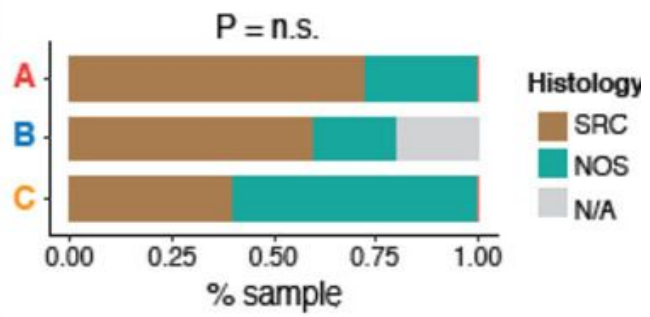

F

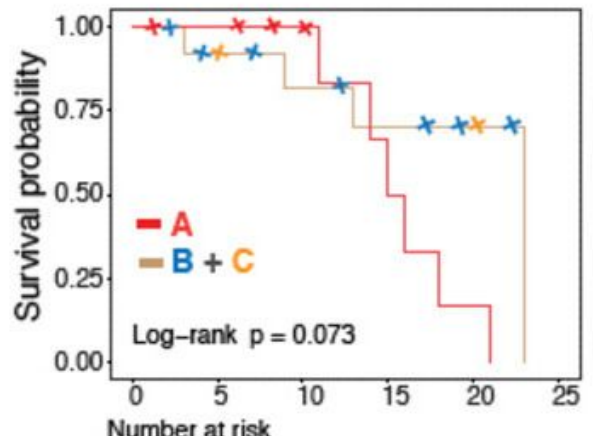

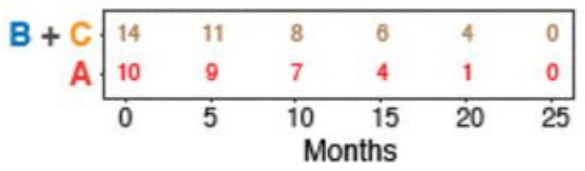

Figure 2

Proteogenomic characterization defined 3 subgroups with differential tumor and immune cell composition. A) Proteomic profiling of 3 subgroups based on tumor and immune cell composition. The cell specific markers for tumor (e.g. EPCAM, MUC1, CDH1, YAP1, ERBB2, KRT8) and immune cell (PTPRC) compartments were used for sample classification and expression of these markers are shown in the top tracks. Heatmap shows differentially expressed genes across 3 subgroups. B) The OncoPrint plot shows 
somatic genomic alterations identified by whole-exome sequencing (WES). CNA, copy number alteration. C) Somatic DNA copy number alterations (CNAs) identified across 22 chromosomes by WES. The frequency of each CNA is labelled on the right axis. D) Copy number gains and losses among cluster $A$ (red), B (blue) and C (orange). The box plot (black) in the violin represent the interquartile range (IQR) and median value. The points in the violin plot depicts the samples in each group. E) Fraction of histological subtypes in 3 defined subgroups. SRC, signet ring cell carcinoma; NOS, non-signet ring cell carcinoma. n.s., not statistically significant. N/A, not available. F) Kaplan-Meier curve shows the difference in patient survival between patients in subgroup $A$ and the rest of patients from subgroups $B$ and $C$. The log-rank test was used to calculate the $p$ value. 
A
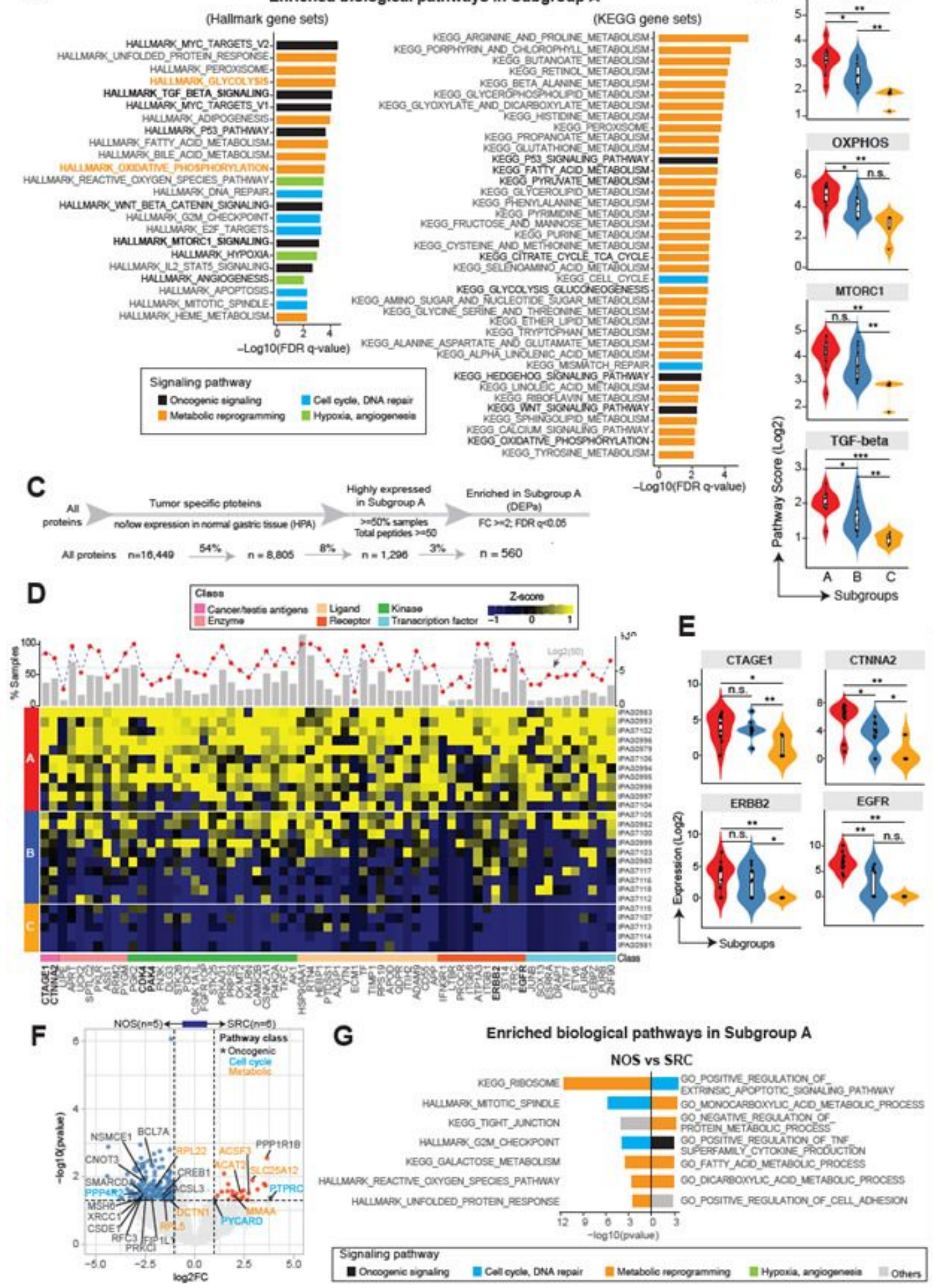

B

(KEGG gene sets)

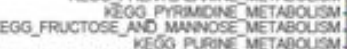

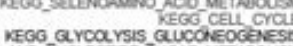

KEGG YEDGEHOG SRGWLVIG PATHWAY

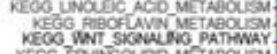

KESG SPH WOLPO METhaOUS

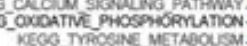

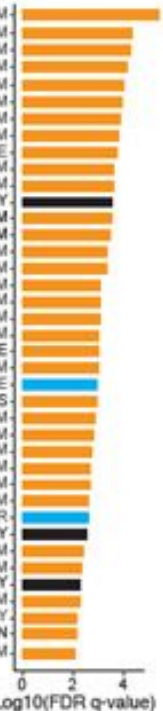

-Log10(FDR Q avalue)

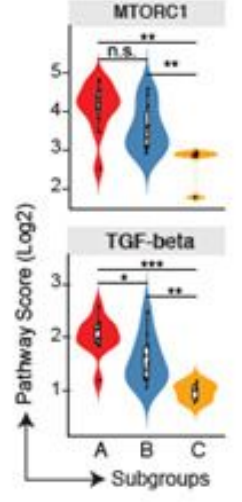

E
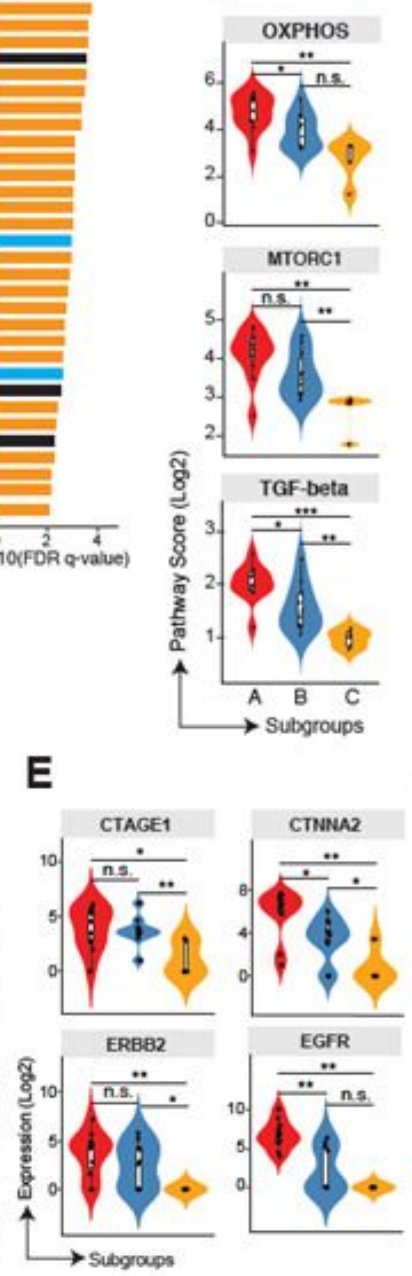

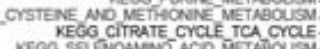

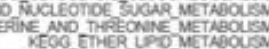

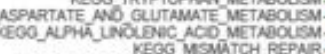

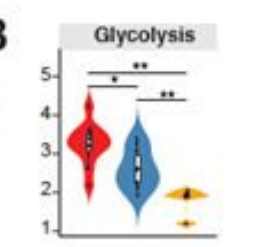

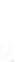


(1\% FDR) is applied. B) Violin plots of representative pathways from the panel A. C) Schematic flow chart showing the filtering process of identifying tumor-specific proteins enriched in the Subgroup A. The proportion and number of proteins retained during each filtering step are shown. D) A heatmap of highly expressed proteins that are enriched in the Subgroup A from the TCE approach. A list of 65 (out of 560) proteins with known functions are shown. The expression values are z-score transformed. Subgroup classification is annotated on the left, sample names are labelled on the right, and protein names are shown at the bottom, with an annotation track color coded by the protein functional category. Top histogram shows for each listed protein, the fraction of samples with detected protein expression by the TCE approach ( $\mathrm{n}=26$ samples). The red filled circle indicates the mean expression level of each protein averaged across 26 samples (y axis on the right, Log2 transformed). The gray dotted horizontal line annotated the expression level of 50 total peptides. E) Violin plots of representative proteins from the panels $D$. The Mann-Whitney $U$ test was used to calculated the $p$ values for data displayed in panels $B$ and $E$. ${ }^{*} p<0.01 ;{ }^{*} p<0.05$; n.s., not statistically significant. F) Volcano plot shows the DEGs between two NOS and SRC with a cutoff of log2(fold change) $>1$ and p-value $<0.05$, different colors indicate the significant genes involving in oncogenic (black), cell cycle (light blue) and metabolic (orange) pathways. G) Pathway enrichment analysis identified biological pathways enriched in NOS and SRC subtypes. The pathways are colored by their biological functions. 
A

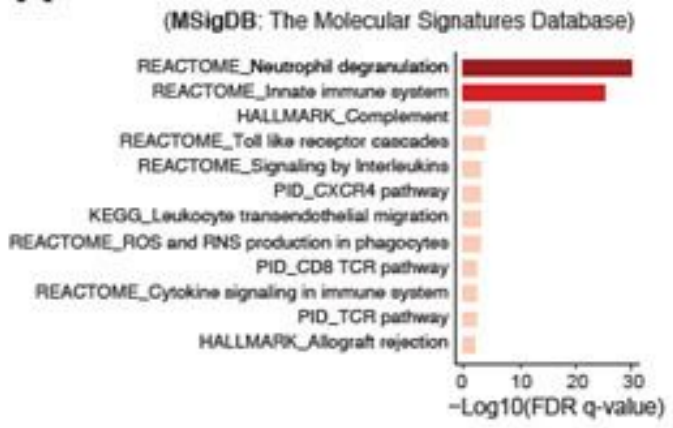

(GO: The Gene Ontology Biological Processes)

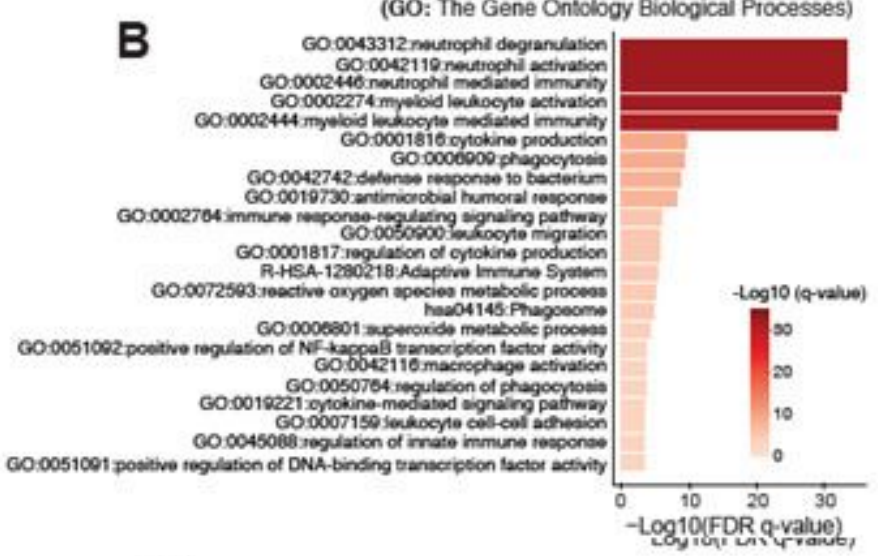

D

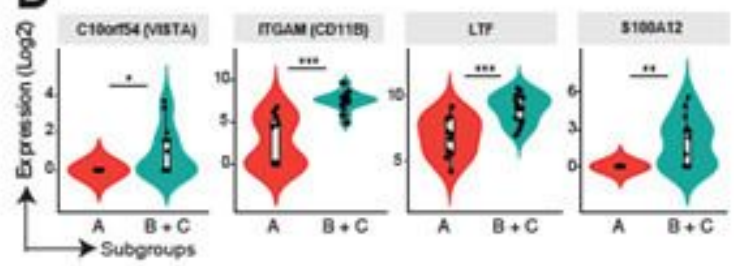

$\mathbf{F}$

Enriched biological pattways in Subgroup B+C NOS subtype

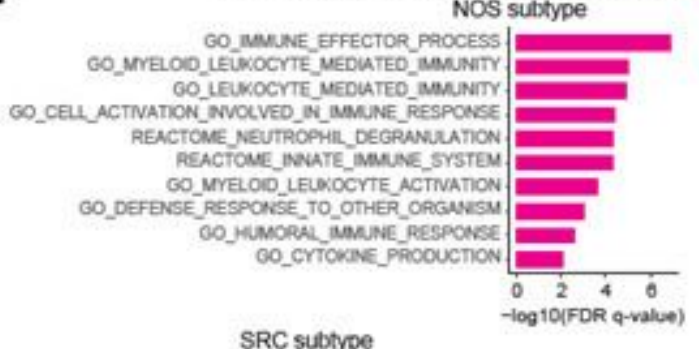

SRC subtype
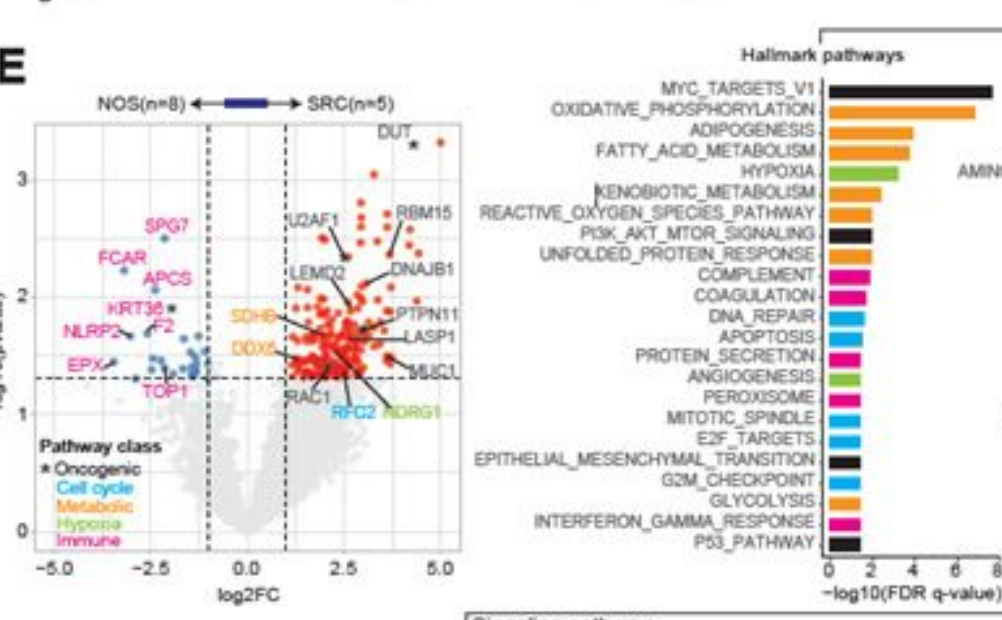

CITRATE, CrCLE, ICA CrCL
FATTY ACID NETABOUSA

MV NUCLEOTOE, SUGAR NETADOUSM

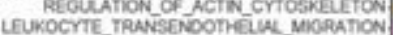

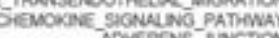

COCOATVE PHOSPHORYATION.

ReOSOW

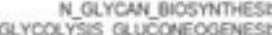

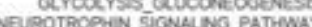

ADPOCYTOKANE- SIGNWLNG PATTEWAY,

PPAR_ SIGNALNG PATHWAY

BIOSWNTHESS_OF_UNSATURATED FATTY ACCDS

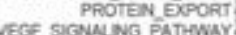

PEAOXISONE

ECU_RECEPTCR NTERACTION APOPTOS

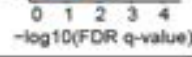

Signaling pathway

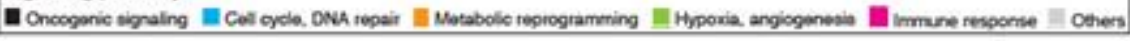

\section{Figure 4}

Enriched TCE proteins and associated biological pathways in the immune-cell-enriched subgroup $\mathrm{C}$ and potential targets. A, B) Pathway enrichment analysis identified biological pathways enriched in the Subgroup $\mathrm{C}$. The curated gene sets were downloaded from the MSigDB and GO databases. FDR q-value, the $p$-value adjusted for the false discovery rate (FDR). A q-value threshold of 0.01 (1\% FDR) is applied. The pathways are colored by their q-values. C) Heatmap of highly expressed proteins that are enriched in the Subgroup C from the TCE approach. Proteins that were highly expressed and significantly enriched in 
the Subgroup C are shown. The expression values are $z$-score transformed. Subgroup classification is annotated on the left, sample names are labelled on the right, and protein names are shown at the bottom. Top histogram shows for each listed protein, the fraction of samples with detected protein expression by the TCE ( $n=26$ samples) approach. The red filled circle indicates the mean expression level of each protein averaged across all samples (y axis on the right, Log2 transformed). The gray dotted horizontal line annotated the expression level of 50 total peptides. D) Violin plots of representative proteins from the panels $C$. The Mann-Whitney $U$ test was used to calculated the $p$ values. ${ }^{*} p<0.01$; ${ }^{*} p<0.05$; n.s., not statistically significant. E) Volcano plot shows the DEGs between two NOS and SRC with a cutoff of $\log 2$ (fold change) $>1$ and FDR q-value $<0.05$ in group $B$ and $C$, different colors indicate the significant genes involving in oncogenic (black), cell cycle (light blue), metabolic (orange), Hypoxia (light green) and immune (pink) pathways. F) Pathway enrichment analysis identified biological pathways enriched in NOS and SRC subtypes in group B and C. The pathways are colored by their biological functions. 

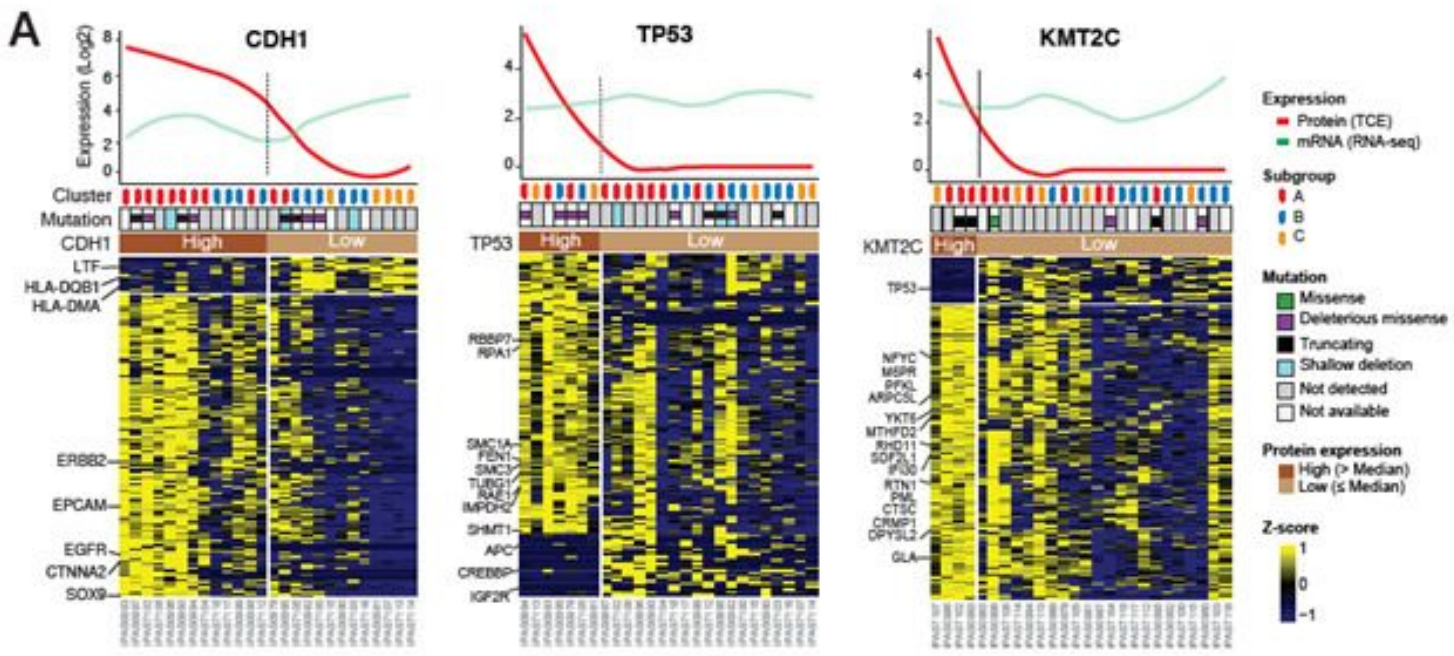

B
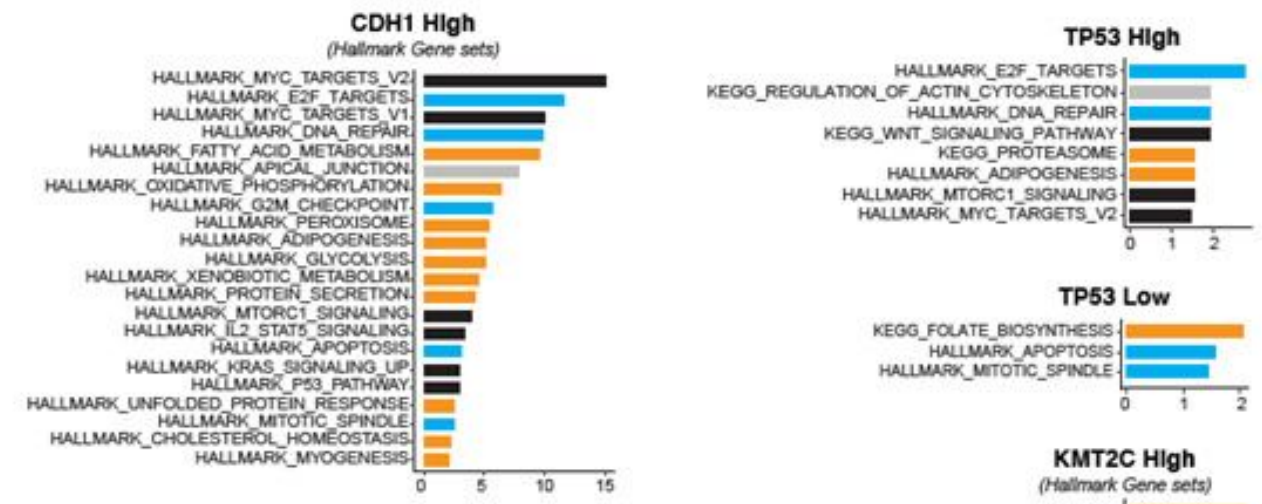

TP53 Low
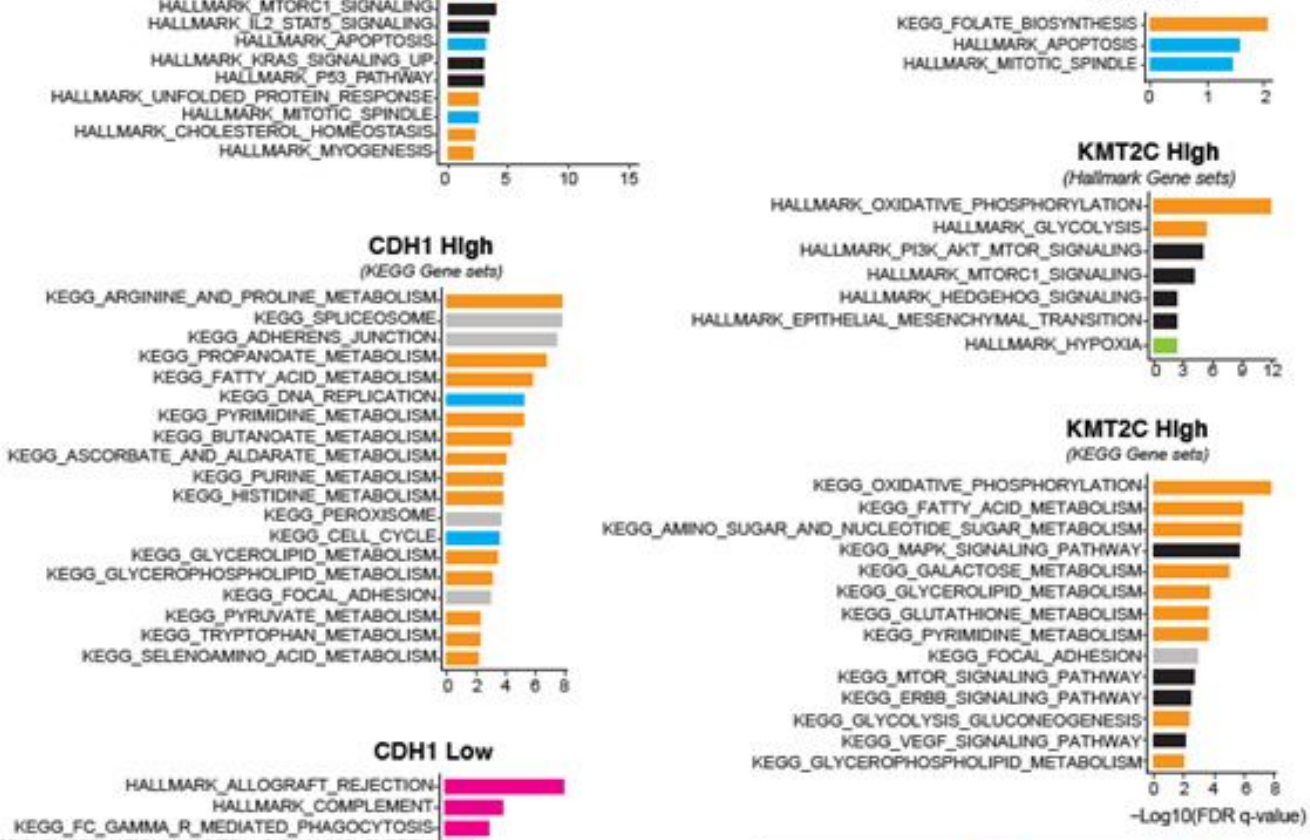

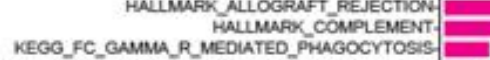

CDH1 LOW

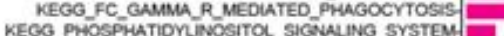

\section{Figure 5}

Distinct protein expression features and signaling pathways associated with aberrant expression of $\mathrm{CDH1}$, TP53, and KMT2C. A). Heatmap shows differentially expressed proteins between the "high" and "low” groups for CDH1 (left), TP53 (middle), and KMT2C (right). The expression values are z-score transformed and data from the TCE approach are shown. The names of some presentative genes are listed. The median expression levels of CDH1, TP53, KMT2C (across 26 samples) were used to define the 


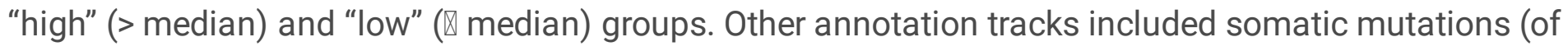
each specific gene) identified from WES, and protein-based subgroup classification from Figure 2 . The curved lines in the top plots indicate the protein and mRNA (mRNA sequencing) expression levels of each specific gene across all samples. B). Differentially expressed signaling pathways between the "high" and "low" groups for CDH1, TP53, and KMT2C.

\section{A}

\section{B}
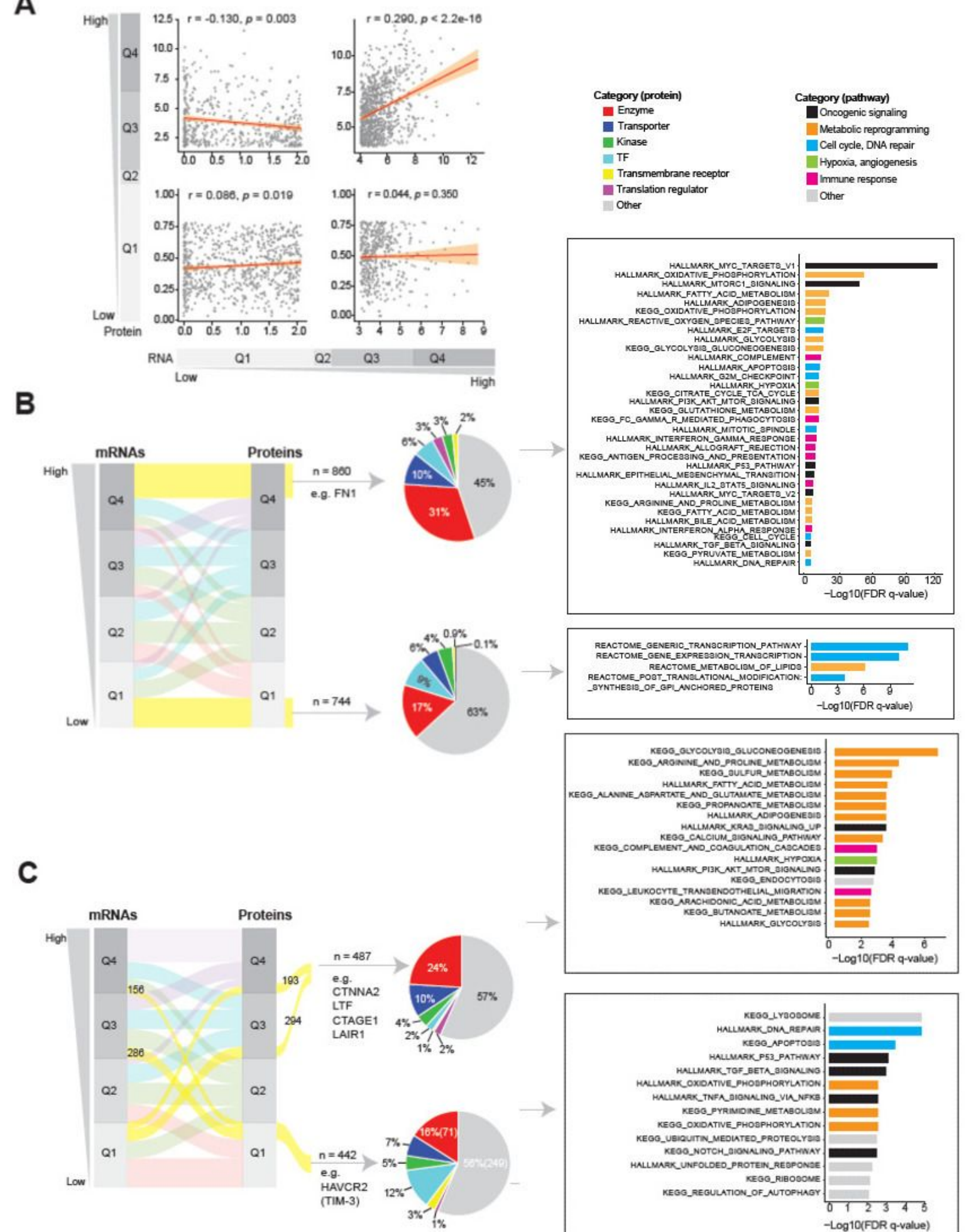

\section{Figure 6}


Correlation between MS protein and mRNA expression and profiling of genes with consistent and distinct expression patterns. A). Correlation analysis between the protein and mRNA expression. The normalized protein expression data from the TCE approach and the normalized mRNA expression data from the whole transcriptome sequencing (RNA-seq) were used for correlation analysis. The correlation analysis was stratified by the protein and mRNA expression levels (protein low and mRNA low; protein high and mRNA high; protein low and mRNA high; protein high and mRNA low). The Pearson's correlation is applied and the correlation coefficient ( $r$ ) and $p$ values are labelled. B). Characterization of genes with consistent mRNA and protein expression. The genes were classified into 4 groups (Q1-4) based on their overall distribution of mRNA and protein expression, respectively. The alluvial plot shows the relationship between protein and mRNA expression for genes in each group and the genes that are consistently high $(n=860)$ or low $(n=744)$ in their expression from both platforms are highlighted (yellow strips) and taken into subsequent analysis, including protein functional categories and pathway enrichment analysis. C). Characterization of genes with inconsistent mRNA and protein expression. The genes were classified into 4 groups (Q1-4) based on their overall distribution of mRNA and protein expression, respectively. The alluvial plot shows the relationship between protein and mRNA expression for genes in each group and the genes that are inconsistently expressed high are highlighted and taken into subsequent analysis, including protein functional categories and pathway enrichment analysis.

\section{Supplementary Files}

This is a list of supplementary files associated with this preprint. Click to download.

- SupplementaryFigure1.pdf

- SupplementaryFigure2.pdf

- SupplementaryFigure3.pdf

- SupplementaryTable1.Characteristicsof26ascitessampleswithgastricadenocarcinoma.xlsx

- SupplementaryTable2.TCE2361Significantproteinsamongthreegroups.xIsx

- SupplementaryTable3.Proteinsdataenrolledinthisstudy.xlsx 